\title{
Hadron melting and QCD thermodynamics
}

\author{
A. Jakovác \\ Institute of Physics, Eotvos University, H-1117 Budapest, Hungary
}

(Dated: August 26, 2021)

\begin{abstract}
We study in this paper mechanisms of hadron melting based on the spectral representation of hadronic quantum channels, and examine the hadron width dependence of the pressure. The findings are applied to a statistical hadron model of QCD thermodynamics, where hadron masses are distributed by the Hagedorn model and a uniform mechanism for producing hadron widths is assumed. According to this model the hadron - quark gluon plasma transition occurs at $T \approx 200-250$ $\mathrm{MeV}$, the numerically observable $T_{c}=156 \mathrm{MeV}$ crossover temperature is relevant for the onset of the hadron melting process.
\end{abstract}

\section{INTRODUCTION}

Hadron Resonance Gas 1] (HRG) provides a good description for a large number of low-temperature QCD observables, as it was demonstrated by a number of studies comparing the result of Monte Carlo (MC) lattice simulations of QCD and the HRG predictions [2 [6]. The strategy of the HRG is quite simple: one takes the particle masses reported by the Particle Data Group [7] and plug them into the ideal gas expression of the pressure. In case of simulations at non-physical masses one has to adjust the hadron masses to the actual numerical environment [4].

The success of the HRG approach, however, raises fundamental questions about the QCD phase transition. The traditional physical reasoning is based on the entropy argument. It states that in the quark gluon plasma (QGP), because of the color of the quarks, the number of degrees of freedom is much larger than that in the hadronic sector, if we count only the stable hadrons (in a 2-flavor case we have 3 pions and 2 nucleons, 3 bosonic and 8 fermionic degrees of freedom, while the 2-flavor QGP contains 8 gluons and $2 \times 3$ quarks, i.e. 16 bosonic and 24 fermionic degrees of freedom). Then the QGP phase, although energetically less favorable, has larger entropy, and thus at high temperature, where the entropy dominates the free energy, this will be the thermodynamic ground state of QCD.

This appealing picture is questioned by the success of HRG, in which all hadrons are treated as thermodynamical degrees of freedom on equal footing. To be more precise, to describe the pressure measured by Monte Carlo (MC) simulations [5] up to the reported crossover temperature $T_{c} \approx 156 \mathrm{MeV}$ [8], one has to take into account $\mathcal{O}(1000)$ hadronic resonances, meaning that in the hadronic phase the effective number of degrees of freedom at this temperature is of this order. But if it is so, the hadron phase is not just energetically favorable, but also has larger entropy, and then no phase transition would be allowed.

It would help if, because of deconfinement, the hadron phase became unstable at a certain temperature, as it was suggested by Hagedorn [9]. But, according to MC results, the transition at $T_{c}=156 \mathrm{MeV}$ is a smooth crossover, and so there is no room for such a violent process like the collapse of a phase. Another hint that the hadron phase will not be unstable above $T_{c}$ is that one can identify the hadronic states at $T>T_{c}$, even up to $T \sim 1.5-2 T_{c}$ [10 14]! In a recent MC study [15] the authors state that the crossover temperature is significantly different for strange and non-strange hadrons, thus there can not be any specific temperature where the hadron phase collapses. All this suggests that the hadrons are present above $T_{c}$ - with their huge entropy factor which forbids a phase transition! Therefore the question is that what happens at $T_{c}$ ?

To be able to answer this question one has to study the hadrons, since they give the dominant part of the entropy at this temperature. There are several facts warning us that the original HRG description is not enough at these temperatures [4]. The most pertinent property of the naive HRG is that after a certain temperature (about 200 $\mathrm{MeV}$ ) it starts to seriously overestimate the real pressure. Strangeness correlations also cannot be described correctly above $T=150-170 \mathrm{MeV}$ (depending on the correlator) with the uncorrelated HRG model [6, 15]. Moreover, a weekly interacting gas would yield large (dimensionless) transport coefficients, in contrast to the reported small value of $\eta / s$ [16].

It is clear that HRG is the simplest representation of the hadron spectrum, in reality it expresses much richer structures than just stable hadrons. Most prominently the hadrons are quasiparticles, and have a finite width, typically of the order $\mathcal{O}(100) \mathrm{MeV}$. Moreover the hadron spectrum contains the continuum of scattering states of other hadrons and eventually of quarks. These properties can be important, in principle the complete spectrum may influence the thermodynamics. For example we expect that hadron width increases with temperature, and finally the hadrons will disappear in the continuum of scattering states: the hadron melts.

There exist different approaches in the literature to take into account the effect of the hadronic width for thermodynamics $17-19]$ and also in transport phenomena 20 22]. These approaches use the approximation that all hadronic resonances are independent. This assumption, although correct for well separated particle peaks [23], gets 
into trouble when the quasiparticle peaks in a given quantum channe1 1 start to overlap, or when a peak is too close to a multi-particle threshold. In the S-matrix approach this is discussed in Refs. 24, 25]. To understand the problem qualitatively, we recall that a quasiparticle is a collective mode, i.e. it is a mixture of the original one-particle energy level and various (actually infinitely many) multiparticle configurations. If two peaks overlap, both quasiparticles would contain the states in the overlapping region. Then, if we performed calculations with two independent quasiparticles, we would overcount the states in the common region. We encounter a similar situation when a peak is too close to a continuum: the overlap would be counted twice if we assumed that the quasiparticle represents a full thermodynamical degree of freedom. This qualitative picture suggest that in these cases we have to decrease the effective number of degrees of freedom.

The goal of this paper is to give a systematic description of this process, referred to as hadron melting, find its effect on the thermodynamics and finally to give some answer to the above question that "what is going on at $T_{c}$ ". Our investigation is based on a previous study, Ref. [26] where the general, mathematically consistent strategy of treating excitations with arbitrary spectral function was worked out, without separating them in an artificial way into quasiparticles, for determining thermodynamical quantities. Here we try to apply these ideas to QCD.

A big obstacle in front of an accurate description of the hadron gas is that the spectral details are usually not known for the hadronic channels, in particular not in the case of large mass hadrons. But, in fact, for thermodynamics we just need a statistical description of the hadrons. Therefore, in this paper, we use an idealized description, based on the Hagedorn spectrum [9]. In this approach one estimates the density of hadronic states by an exponentially growing function. The original proposal of Hagedorn was $\varrho_{h a d r}(m) \sim\left(m^{2}+m_{0}^{2}\right)^{-5 / 4} e^{m / T_{H}}$, where $m_{0} \approx 500 \mathrm{MeV}$ and $T_{H}$, the Hagedorn temperature, is of the order of $200-300 \mathrm{MeV}$. The validity of this assumption was verified lately [27], where the authors have pointed out that a large variety of exponentially growing functions (in particular a pure exponential) is appropriate to fit the observed hadron spectrum. Similar works prove the plausibility of the Hagedorn-assumption [28]. In an ensemble containing free particles with masses distributed by the Hagedorn distribution, the pressure has a singularity at $T=T_{H}$. This resembles to the fast growth of the pressure in the HRG computations: this is also due to the contribution of the large numbers of massive hadronic states. Therefore, to understand the reduction of the pressure of the real hadron gas, the study of the Hagedorn gas is an excellent tool.

Our strategy is to first study simplified models of melting of a bound state, focusing on the possible reasons of melting: a particle can melt into a continuum because of growing width, and because of decreasing wave function renormalization constant. In principle there can be a third mechanism, when different quasiparticle peaks merge to form a broad continuum. This can be relevant for the Coulomb problem of QED, for example, where infinitely large number of states are present very densely at zero temperature in each quantum channel (eg. in the $s$ channel the $n s$ states have energy $E_{0} / n^{2}$ and width $\sim 1 / n^{3}$ ). But, according to the PDG tables, in QCD in each quantum channel there are at most 2-3 excitation peaks present, so probably the most important source of melting of hadrons is one of the two scenarios mentioned above. The lesson of the model studies will be summarized in a single simple formula, giving the dependence of the pressure of the system on the quasiparticle width, in the presence of a continuum. This is a key point of the paper, cf. (15). The coefficient for the reduction of the effective number of degrees of freedom reads $\sim e^{-c \gamma^{2}(T)}$, where $\gamma(T)$ is the hadron width. This formula makes it possible to describe, how in the course of hadron melting the pressure of the hadronic matter decreases. We find that up to $200-250 \mathrm{MeV}$ one can easily describe the existing MC data even with the simplest purely hadronic fits, but above this temperature, in all parametrizations we used, the hadronic pressure contribution becomes much smaller than the measured one.

This leads us to propose the following answer to the above posed question: $T_{c}=156 \mathrm{MeV}$ is the starting point of rapid hadron melting, and not a hadron - QGP transition. The quark gluon plasma appears at a higher $T_{c}^{\prime}$ temperature; our model calculations propose for it a range of $T_{c}^{\prime} \in[200,250] \mathrm{MeV}$.

The paper is organized as follows: first we shortly overview the consequences of the Hagedorn spectrum for the energy density and pressure, and recall the most important results of Ref. [26]. Next we will consider the two simplified models of particle melting. We study the energy density and pressure in these models, and in particular we determine the effective number of degrees of freedom, i.e. the pressure reduction factor. Then we apply the findings to QCD thermodynamics, and present our fit to the results of the MC measurements. We close the paper with our Conclusions.

\footnotetext{
${ }^{1}$ Quantum channel in this work means superselection class, i.e. states having the same quantum numbers.
} 


\section{PARTICLE DISTRIBUTION AND SPECTRAL FUNCTIONS}

If we have free bosonic/fermionic particles with masses $m_{n}(n=1 \ldots N)$, then the pressure of this mixture (at zero chemical potentials) reads in thermal equilibrium

$$
P_{\alpha}=-\alpha \frac{T}{2 \pi^{2}} \sum_{n=1}^{N} \int_{0}^{\infty} d p p^{2} \ln \left(1-\alpha e^{-E\left(p, m_{n}\right) / T}\right)
$$

where $\alpha= \pm 1$ for bosons/fermions, and $E(p, m)$ is the dispersion relation, for relativistic case $E^{2}=p^{2}+m^{2}$.

For a large number of nearby mass values we can introduce the (hadronic) mass density function $\varrho_{h a d r}$ with the definition

$$
\varrho_{h a d r}\left(m_{n}\right)=\frac{1}{m_{n+1}-m_{n}}
$$

This makes possible to approximate the above formula as an integral, formally inserting $d m \varrho(m)=1$ and apply Riemann integral formula

$$
P_{\alpha} \approx-\alpha \frac{T}{2 \pi^{2}} \int_{0}^{\infty} d m \int_{0}^{\infty} d p \varrho_{h a d r}(m) p^{2} \ln \left(1-\alpha e^{-E(p, m) / T}\right)
$$

For massive hadrons $m \gg T$ we can use nonrelativistic approximation $E=m+p^{2} /(2 m)$, and $e^{-E / T} \ll 1$. Then we find

$$
P \approx \frac{T^{5 / 2}}{(2 \pi)^{3 / 2}} \int_{0}^{\infty} d m \varrho_{h a d r}(m) m^{\frac{3}{2}} e^{-m / T}
$$

In the case of Hagedorn spectrum the hadronic density function grows exponentially $\varrho_{h}(m) \sim e^{m / T_{H}}$, and the integral is divergent for $T>T_{H}$. This would signal, according to the original argumentation of [9], a thermodynamical instability of the hadron gas. This scenario, however, is manifested in QCD in a different way.

For numerical computation we should use the discrete mass summation of eq. (11). For simplicity we apply a purely exponential hadron mass density $\varrho_{h a d r}$ which corresponds to the following hadron mass series

$$
m_{n}=M+T_{H} \ln n
$$

The parameters $\left(M\right.$ and $\left.T_{H}\right)$ of this formula can be chosen to fit the pressure data from Monte Carlo (MC) simulations. In Fig. 1 we used 5000 bosonic resonances to fit the data of the BMW group [5] for the pressure and the interaction measure or trace anomaly $(I=\varepsilon-3 p)$, both scaled by $T^{4}$. The fit parameters turned out to be $M=120 \mathrm{MeV}$ and

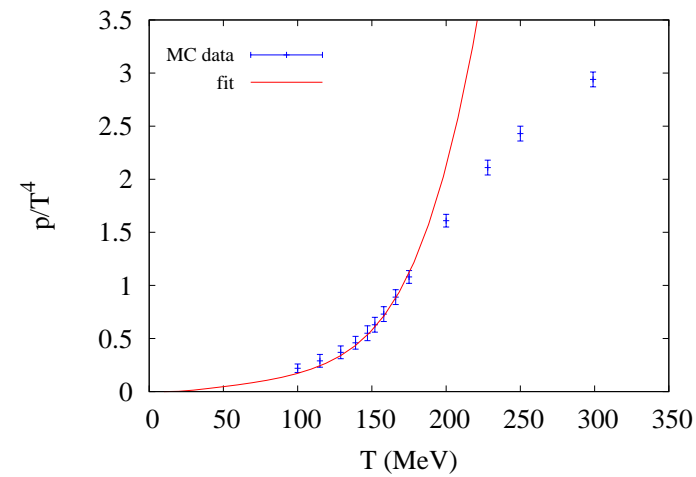

a.)

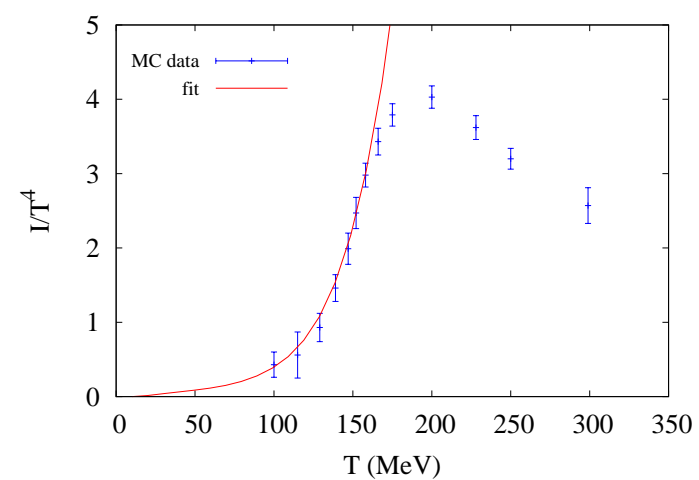

b.)

FIG. 1. Hagedorn fits to the MC pressure data (a.) and interaction measure $(I=\varepsilon-3 p)$ data (b.) from [5]. The solid (red) line is the result of 5000 particles with Hagedorn mass distribution (5) with parameters $M=120 \mathrm{MeV}$ and $T_{H}=240 \mathrm{MeV}$. 
$T_{H}=240 \mathrm{MeV}$. Both are reasonable values, $M$ is close to the pion mass 2 and a reasonable Hagedorn temperature value. It is remarkable that even with this simplest mass density formula we obtain excellent fits at lower temperatures. Since the number of excitations is finite, we do not have a real singularity at $T_{H}$, though the value of the corresponding $\mathrm{SB}$ limit is still about 50 times the SB value of QCD.

In the above analysis we have only taken into account the free gas description of the hadronic matter. The high mass resonances, however, usually have very large width, often in the range of $\mathcal{O}(100) \mathrm{MeV}$ which is temperature dependent, and they are close to the continuum formed by hadronic scattering states and - at higher temperatures by quark-gluon scattering states. So, in particular at growing temperature, sooner or later we inevitably get into the regime when the resonances in a given quantum channel overlap with each other and with the multiparticle continuum. Then, as it was discussed in the introduction, the free gas approximation significantly overestimates the real pressure, since the overlapping regimes are counted more than once.

The correct treatment of a system described by a generic spectral function $\varrho_{S}(p)$ was worked out in [26]. The key result of that paper is that the energy density and pressure coming from a bosonic excitation should be calculated by the formulae

$$
\varepsilon=\int \frac{d^{4} p}{(2 \pi)^{4}} \varrho_{S}(p) \mathcal{H}(p) \Theta\left(p_{0}\right) n\left(p_{0}\right), \quad P=-T \int \frac{d^{4} p}{(2 \pi)^{4}} \varrho_{S}(p) \mathcal{H}(p) \frac{\Theta\left(p_{0}\right)}{p_{0}} \ln \left(1-e^{-\beta p_{0}}\right),
$$

where $n\left(p_{0}\right)=\left(e^{\beta p_{0}}-1\right)^{-1}$ is the Bose-Einstein distribution, and (with a principal value integration denoted by $\mathcal{P}$ ):

$$
\mathcal{K}^{-1}(p)=\mathcal{P} \int \frac{d \omega}{2 \pi} \frac{\varrho_{S}(\omega, \mathbf{p})}{p_{0}-\omega}, \quad \mathcal{H}(p)=p_{0} \frac{\partial \mathcal{K}}{d p_{0}}-\mathcal{K} .
$$

An important property of (6) is that the thermodynamical quantities are insensitive to the normalization of the spectral function, since $\mathcal{K}$ and thus $\mathcal{H}$ is inversely proportional to $\varrho_{S}$. Therefore, although the proper magnitude of $\varrho_{S}$ is determined by the sum rules, its actual value does not influence thermodynamics.

Technically one arrives at these formulae by introducing an effective quadratic theory with kernel $\mathcal{K}$ which reproduces the input spectral function $\varrho_{S}(p)$. Then one calculates the energy-momentum tensor (Noether-current), and takes the finite temperature expectation values. The equations are quite plausible, for example the energy density $\varepsilon$ sums up, for all spatial momenta, all excitation energy levels with a Bose-Einstein distribution, associating $\mathcal{H}$ energy density contribution to them. The distinction relative to the naive formula is that here the associated energy density also depends on the spectral function in a nontrivial way. Heuristically, $\mathcal{H}$ is related to the kernel of the quadratic effective theory $\mathcal{K}$, like the Hamiltonian is related to a Lagrangian in an ordinary mechanical system, if $p_{0}$ replaces the time derivative of the coordinate.

It was shown in [26] that if $\varrho_{S}$ consists of Dirac-delta peaks

$$
\left.\varrho_{S}(p)\right|_{\text {free }}=\sum_{n=1}^{\infty} Z_{n} \delta\left(p^{2}-m_{n}^{2}\right)
$$

then eq. (6) reproduces the usual ideal gas energy density of independent free particles. It is remarkable that in this limit the thermodynamics is independent of the individual normalizations $Z_{i}$. This, of course, will not be true any more in case of overlapping peaks, it is only invariant under an overall normalization of the spectral function.

\section{MODELS OF HADRON MELTING}

Now the task is to go beyond the ideal gas description of (8), and try to incorporate the width and the threshold effects into the spectral function. For a realistic description we would need a detailed knowledge of the hadronic spectral functions at finite temperature. This is not available yet, and probably it will be not available in the near future. Therefore here we consider two clean model-scenarios, corresponding to different mechanisms of hadron melting. In both cases we will study the thermodynamics of the system.

In these scenarios we consider a spectral function describing a quasiparticle peak at mass $m$ and a multiparticle continuum. The nonzero value of the continuum at the quasiparticle energy yields a finite width for the quasiparticle. The most general setup would have been a model consisting of a finite number of bound states with different width

\footnotetext{
${ }^{2}$ In Hagedorn spectrum all hadrons participate without multiplicity factor, so real pions should be distributed at three different mass values.
} 
parameters, and a multiparticle continuum. According to the PDG particle tables, however, in most quantum channels there is only one observed bound state, in particular in the higher mass regions, but also in case of smaller masses there are just a few (2-3). Therefore, the simplest and quite realistic approach is to study the thermodynamics when a single bound state peak overlaps with the continuum states.

In one of the scenarios we will vary the width of the quasiparticle. At zero temperature it can be even zero if the threshold of the continuum $m_{t h}$ is larger than the particle mass $m$. This describes a stable particle, it then represents one thermodynamical degree of freedom. As the temperature increases, a nonzero value of the spectral function develops below the zero temperature threshold which yields nonzero width for the bound state. Finally, when the width is large, the bound state peak melts into the continuum. Physically this situation can be the most relevant when studying bound states near the threshold. Note, that when the width is large enough, the bound state is not identifiable at all, it completely dissociates into its components forming the continuum.

In the other scenario we consider a bound state which may have a small width, but the main process is the decrease of the wave function renormalization of the peak. This can happen because the multiparticle continuum increases by temperature effects. Because of the sum rule this must be accompanied by a shrinking quasiparticle wave function renormalization. As a result, the particle sinks into the continuum, and disappears from the thermal medium, even when its width is not changing too much. In this case the quasiparticle remains identifiable, just its thermal weight gets smaller. This situation is manifested in usual chemical reactions, when all reagents keep their (quasi)particle nature, just their concentration changes.

In the melting of hadrons probably both effects are present. In MC simulations [13] one can clearly see the decreasing height of bound state peaks (e.g. in $J / \Psi$ channel), but the spectral representation is not precise enough to make distinction between the two possible scenarios.

For both scenarios, the quasiparticle peak is represented by a Lorentzian:

$$
\varrho_{L o r}(p)=\frac{4 h \gamma^{2} p^{2}}{\left(p^{2}-m^{2}\right)^{2}+4 \gamma^{2} p^{2}} .
$$

In this parametrization $h$ is the height of the peak. Near $p^{2}=m^{2}$ mass shell the above formula behaves as

$$
\varrho_{L o r}(p=m+x) \approx \frac{\zeta \gamma}{x^{2}+\gamma^{2}}, \quad \zeta=h \gamma,
$$

and so $\gamma$ is the peak width, $\zeta$ is the wave function renormalization constant.

The continuum is modeled by a 2-particle spectral function, but with imaginary threshold mass (in order to mimic its value under the threshold value which is responsible for the width of the quasiparticle peak), and a correction prefactor to improve the $p \rightarrow 0$ behavior:

$$
\varrho_{\text {cont }}(p)=\frac{p}{p^{2}+m_{t h}^{2}} \operatorname{Im} \sqrt{m_{t h}^{2}+i S-p^{2}}=\frac{p}{p^{2}+m_{t h}^{2}} \sqrt{\frac{\sqrt{\left(p^{2}-m_{t h}^{2}\right)^{2}+S^{2}}+p^{2}-m_{t h}^{2}}{2}} .
$$

Its normalization is chosen to be unity, therefore the quasiparticle $\zeta$ is a relative wave function renormalization. The complete spectral function is $\varrho=Z\left(\varrho_{L o r}+\varrho_{\text {cont }}\right)$, where the value of $Z$ is responsible for satisfying the sum rule. Then the complete wave function renormalization of the quasiparticle peak is $\zeta Z$. But $Z$, being an overall normalization factor, drops out from thermodynamical observables, as it was discussed above.

In the present paper we used $m=1$ and $m_{t h}=2$ values. $S$ is determined by the condition that the value of the continuum at $m$ is $\gamma$. In the first scenario we keep $\zeta$ fixed and increase the peak width, in the second scenario we keep $\gamma$ fixed, and decrease the peak height.

First let us look at the result of the first scenario. For various $\gamma$ values we can see the shape of the spectral functions 3 in Fig. 2/a. We see that the peak has larger and larger width which results in the suppression of the separation between the peaks and the continuum. Still, the peak stays clearly identifiable.

After evaluating the integrals of (6), the corresponding pressure data can be seen in Fig. 2/b. We see, how in the course of increasing the overlap between the bound state and the continuum the pressure decreases. We can observe that, despite the fact that the peak cannot be missed in the spectrum, it is not a thermodynamical degree of freedom beyond a certain width. Considering the two widest peaks of the spectrum in Fig. 2/a, for $\gamma=0.045$ we have half of the pressure yet, for $\gamma=0.137$ the pressure practically vanishes. The rule of thumb which can be deduced from here is similar to the findings of [26]: if $\gamma / \Delta m$ exceeds about $10 \%$, the peak cannot be considered as a standalone, ideal thermodynamical degree of freedom, although dynamically (eg. in linear response theory) one still can identify it.

\footnotetext{
${ }^{3}$ Note that the curves are rescaled for better visibility
} 


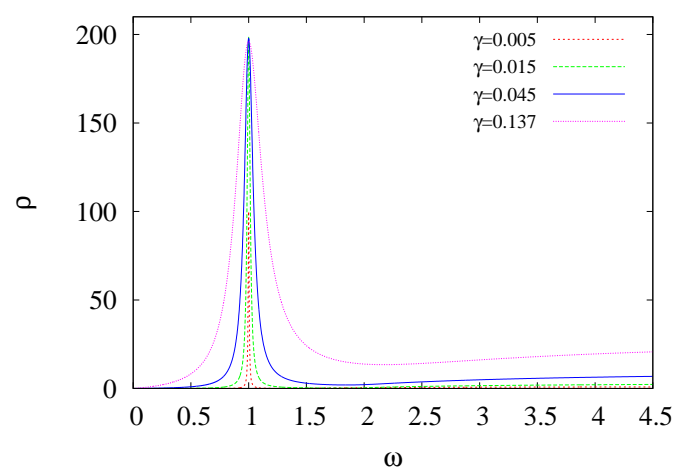

a.)

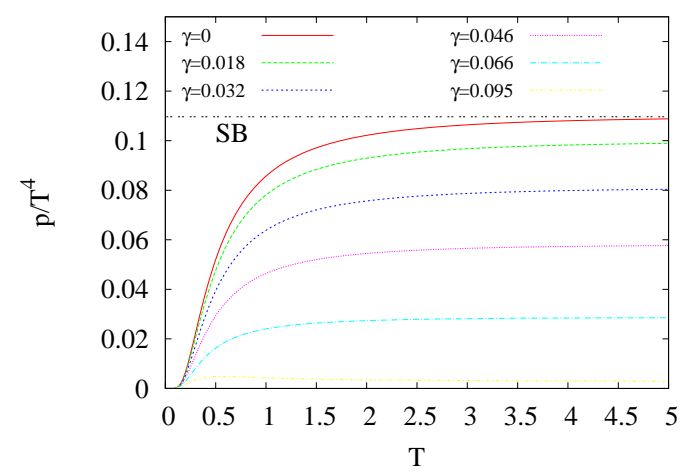

b.)

FIG. 2. a.) Spectral functions consisting of a single peak with different width parameters at $m=1$ and a 2 -particle cut starting at threshold $m_{t h}=2$. The curves are rescaled for better visibility. b.) The width dependence of the pressure.

In case of the second scenario we consider spectral functions plotted in Fig. 3/ a. Here all plots contain a quasiparticle with the same width $(\gamma=0.05$ in the present case), but with decreasing relative peak height (the plots are rescaled to have a constant peak height with increasing value of the continuum). We see that although the peak width is constant, the overlap is still getting larger because of the increasing relative weight of the continuum part.

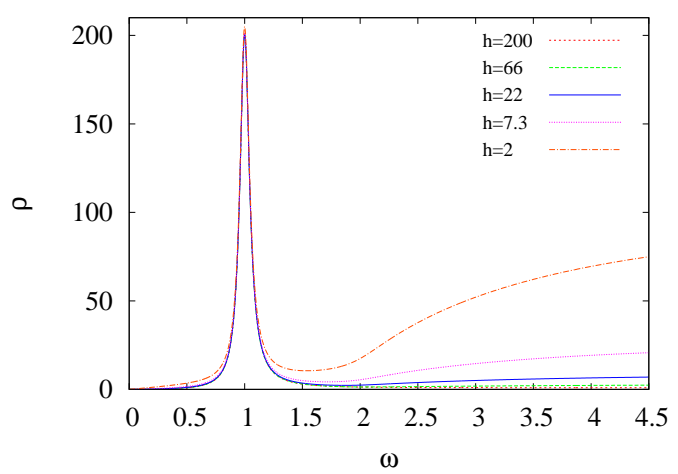

a.)

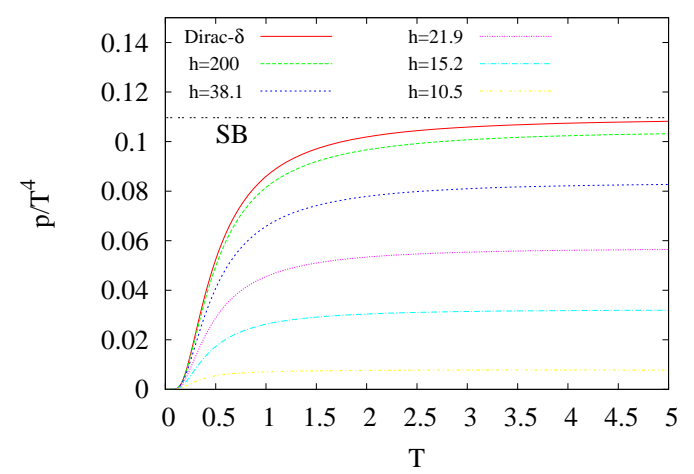

b.)

FIG. 3. a.) Spectral functions consisting of a single peak at $m=1$ and $\gamma=0.05$, and a 2-particle cut with varying relative height starting at threshold $m_{t h}=2 . h$ is the peak height of (9). The curves are rescaled to keep the quasiparticle peak unchanged. b.) The dependence of the pressure on the continuum height.

The pressure computed from these spectral functions, using ([6), can be seen in Fig. [3/b. The curves are very similar to the previous scenario: increasing relative continuum height leads to decreasing pressure. We can observe also here the fact that, even when the peak is clearly identifiable in the spectrum, and thus dynamically it still dominates the long time linear response, in thermodynamics its contribution is already negligible.

We should emphasize that we have tried several parameter sets in the aforementioned scenarios and also various shapes for the spectral functions. The qualitative behavior, however, still remained the same as in these cases.

\section{A. Effective number of thermodynamical degrees of freedom}

Our goal, of course, is to tell something about the thermodynamics of QCD. To this end we want to draw some robust consequences of the discussed toy model calculations which are independent of the detailed mechanisms.

A common feature of all cases was the reduction of the number of thermodynamical degrees of freedom. As we have seen, this quantity is not closely related to the observability of the quasiparticle peaks in the spectrum, thermodynamically the quasiparticle excitations become much earlier negligible. Therefore, we introduce the effective number of thermodynamical DoF $N_{\text {eff }}$ which depends on the temperature and the quasiparticle parameters (width, 
height), and is defined simply as

$$
N_{\text {eff }}(T)=\frac{p(T)}{p_{\text {ideal }}(T)},
$$

where in the ideal case we have only a Dirac-delta peak with mass $m=1$. Evaluating this expression for our modelscenarios resulted in plots of Fig. 4. The plots are restricted to the physically sensible positive pressure parts. The

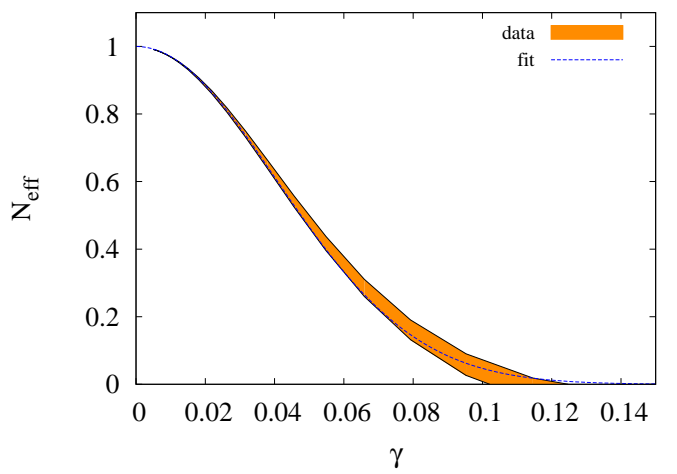

a.)

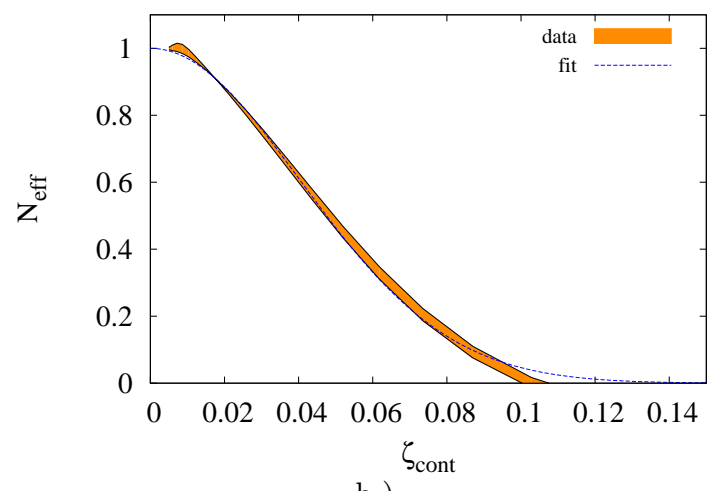

b.)

FIG. 4. Effective number of thermodynamical degrees of freedom in the studied temperature range, for a.) Scenario 1, b.) Scenario 2. The shaded regions represent temperature dependence of the definition. The fit functions were Gaussian.

control parameter in case of Scenario 2 was

$$
\zeta_{\text {cont }}=\frac{1}{1+h}
$$

where $h$ is the peak height. $1-\zeta_{\text {cont }}$ can be physically interpreted as a quantity which is proportional to the wave function renormalization of the quasiparticle peak.

The definition of $N_{\text {eff }}$ is, in principle, temperature dependent, so if we plot it against $\gamma$ or $\zeta_{\text {cont }}$ we have several values corresponding to the actual temperature choice. The shaded regions in Fig. 4 show the temperature variation of $N_{\text {eff }}$. As we see it is rather moderate, and it makes it possible to speak about the number of thermodynamical degrees of freedom independently of the actual temperature.

We can also try to write up an analytic approximate formula for these numerically determined curves. Surprisingly, it appears that a simple Gaussian curve

$$
N_{e f f}(x)=e^{-x^{2} / 2 \sigma^{2}}
$$

works excellently in both cases, where $x$ is the corresponding control parameter. In the actual calculations in the first scenario the variance was $\sigma_{\gamma}=0.04$, in the second one $\sigma_{\zeta \text { cont }}=0.04$, too (a mere numerical coincidence).

\section{QCD THERMODYNAMICS}

Now let us try to draw the consequences of the above studies for QCD. We emphasize it again that, according to the Hagedorn picture, the thermal properties of QCD are determined by the statistical ensemble of all hadrons. So we have to determine the thermal behavior of an "average" hadron.

The fact that in both of our model-scenarios we found Gaussian reduction factor for the effective number of dof, suggests that in the QCD case we should also take Gaussian trial functions for this quantity. There are two effects which we have to take into account. The first is that the relative weight of the continuum, here characterized by the pure number $\zeta_{\text {cont }}$, increases with the number of the decay channels. For increasing masses, therefore, we should have larger $\zeta_{\text {cont }}$ values. If $\zeta_{\text {cont }}$ is at least linearly proportional to the hadron mass, then from the Gaussian form we find a regularizing factor $\sim e^{-\# m^{2}}$ (or even faster decreasing with $m$ ). This results that the most massive hadronic states will never appear as separate full-fledged thermodynamical degrees of freedom. In our calculation - since the exact form of $\zeta_{\text {cont }}$ is not known - we take this effect into account by introducing a maximal number of hadronic resonances. 
Similar technique is proposed in [29]. We have checked that the final results do not depend too much on the exact choice of this number.

The other effect is the temperature dependence of the width of the bound states. Here we assume that the partial pressure of all hadrons decreases in the same way. Physically we can motivate this choice by the observation that the typical width of the hadrons at zero temperature is in the same range of $\mathcal{O}(100) \mathrm{MeV}$, which makes it probable that the physics which governs the width of the hadrons is similar, too. The fact that the light and strange-quark susceptibilities are rescaled versions of each other [15], also supports the idea of a common melting mechanism.

Then, using (12) and the fit of Fig. 4 we may consider a simple Ansatz

$$
p(T)=p_{H a g}(T) N_{e f f}(\gamma(T))=p_{H a g}(T) e^{-\kappa \gamma^{2}(T)},
$$

where $p_{H a g}$ is the pressure coming from the ideal Hagedorn gas spectrum.

The (average) hadron width function $\gamma(T)$ is not really known, but we can make the following considerations. First of all the width is not zero at $T=0$ for most cases. On the other hand dimensional analysis suggests that at high temperatures $\gamma(T) \sim T$. In many model calculations these two regimes are cleanly separated: for small temperatures the width is nearly constant or just slightly temperature dependent, while at higher temperatures we get into a linearly rising regime. One finds this behavior in the $\Phi^{4}$ model in two-loop 2PI approximation [30], in QCD using sum rules [31], in the holographic approach [32], and it is consistent also with pion gas 2PI calculations [33]. To mimic this behavior we will take the following simple form 4 for the suppression factor

$$
\kappa \gamma^{2}(T)=\kappa_{0}^{2}+\delta \kappa^{2}(T), \quad \delta \kappa(T)=\Theta\left(T-T_{0}\right)\left(T-T_{0}\right) / T_{n} .
$$

Here $\kappa_{0}$ represents the effect of the zero temperature suppression, $T_{0}$ is the temperature where the linear regime starts, $T_{n}$ is connected to the variance of the Gaussian.

There are three parameters in the above formula which are determined by fitting the MC trace anomaly data. Interestingly the fits are quite robust in the sense that it is relatively easy to find reasonably fitting solutions, with rather different values of the parameters. For example, varying the Hagedorn temperature $T_{H}$, the starting mass $M$ and the zero temperature damping $\kappa_{0}^{2}$ all change the curve similarly, and affect mainly the low temperature part of the curve. $T_{0}$ plays a not too important role, and finally $T_{n}$ is determined by the height of the trace anomaly curve. We plot some of the results in Fig. 5. The fit parameters can be seen in Table 1 .

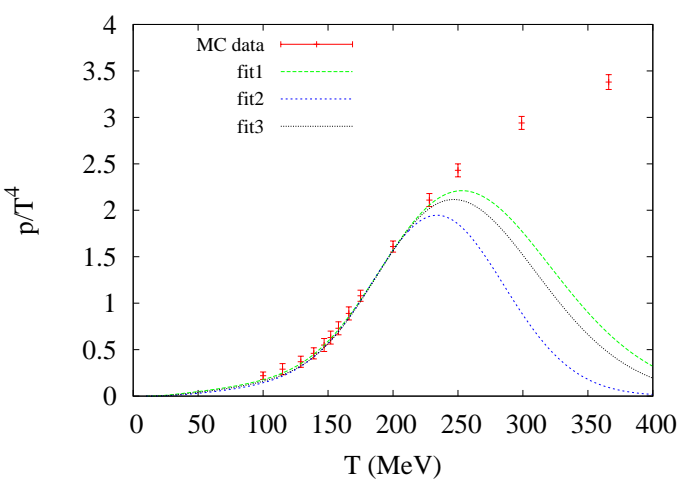

a.)

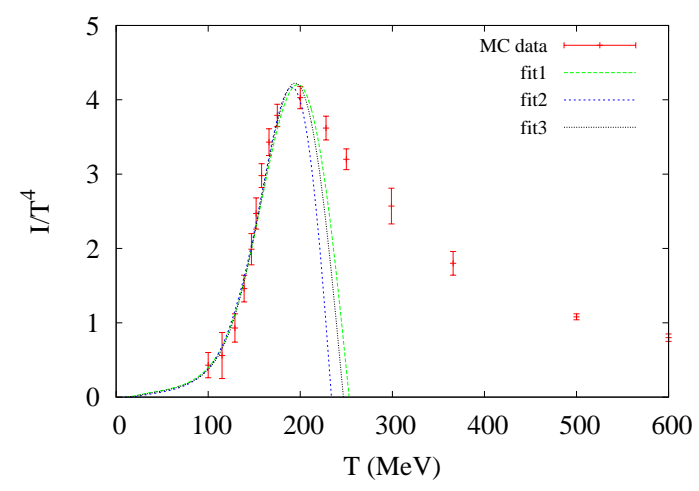

b.)

FIG. 5. Effect of the reduction of the number of degrees of freedom on the Hagedorn spectrum: a.) pressure, b.) interaction measure. The parameters of the fits is contained in [.

\begin{tabular}{||c|c|c|c|c|c|c||}
\hline fit \# & $N$ & $T_{H}(\mathrm{MeV})$ & $M(\mathrm{MeV})$ & $\kappa_{0}^{2}$ & $T_{0}(\mathrm{MeV})$ & $T_{n}(\mathrm{MeV})$ \\
\hline 1 & 20000 & 195 & 115 & 0 & 0 & 158 \\
2 & 20000 & 220 & 140 & 0.2 & 125 & 93 \\
3 & 40000 & 190 & 130 & 0 & 0 & 147 \\
\hline
\end{tabular}

TABLE I. The fit parameters

\footnotetext{
${ }^{4}$ Of course, other parametrizations are also possible, and we tried some of them different formula, too, but the final outcome remained the same.
} 
The first fact that we should pin down is that indeed our effective model is capable to provide a hadronic partial pressure which is smaller than the complete pressure, as we should require.

If we look through the parameter choices of the different fits in Table \, we see that the first and third fits use a minimal setup $\left(\kappa_{0}^{2}=0\right.$ and $\left.T_{0}=0\right)$, the difference between these two cases is the number of hadronic resonances we have taken into account. Thus these fits are pure Gaussian $N_{\text {eff }}(T)=e^{-T^{2} / T_{n}^{2}}$. Moreover, the lightest hadron masses were $M=115 \mathrm{MeV}$ and $130 \mathrm{MeV}$, which are consistent with a smeared out pion mass. Still this very naive approach reproduces nicely the details of both the measured hadronic pressure and the trace anomaly (interaction measure)! The second row of Table $\Pi$ is a typical fit with all parameters involved. We observe just very little difference between the corresponding curves in Fig. 5. We must also emphasize that other thermodynamical quantities can be computed from these two, eg. $\varepsilon=I+3 p$ and $T s=I+4 p$ are the energy density and entropy density, respectively. Therefore, we can state that these curves provide fits for the complete QCD thermodynamics in a certain temperature range.

The most striking property of these curves is that they can describe full QCD pressure and interaction measure up to about $200-250 \mathrm{MeV}$ (depending on the parametrization of the width). Since the full pressure is the sum of the partial pressures of the hadronic and QGP quantum channels $P_{\text {tot }}=P_{\text {hadrons }}+P_{Q G P}$, we can read out the QGP pressure and interaction measure coming from this model. We plotted these quantities in Fig. [6] (using the first row of Table (1). As we can see, the pressure of the QGP disappears below of about $250 \mathrm{MeV}$. The physical reason is the appearance of hadronic bound states which results in a drastic decrease of the mean free path and lifetime of the QGP excitations.

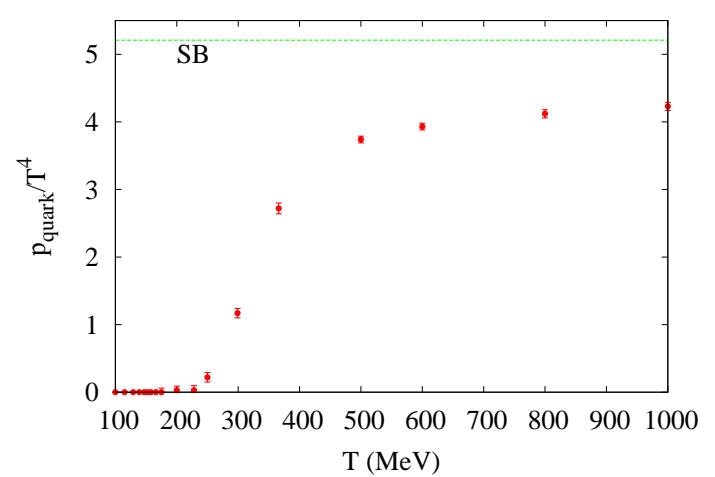

a.)

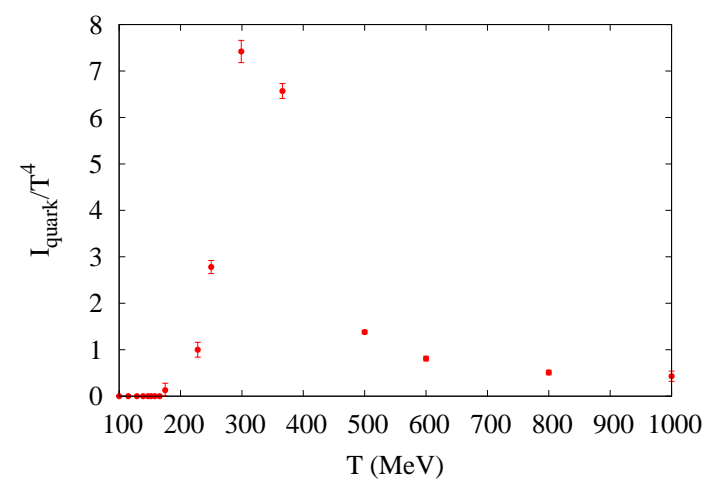

b.)

FIG. 6. The pressure and trace anomaly (interaction measure) of the QGP, according to our model predictions and MC data. SB means the asymptotic Stefan-Boltzmann limit.

\section{CONCLUSIONS}

In this paper we have studied the process of hadron melting based on the method of Ref. [26]. To describe real hadrons in a thermal environment we have considered two model-scenarios for the spectral function which shows the presence of resonances and a continuum in a given quantum channel. The two cases differ in the way of choosing the control parameter. We have found in all cases that increasing the deviation from the single stable particle spectral function resulted in a decreasing pressure, i.e. the quasiparticle, represented by the peak "melts". The effective thermodynamical dof, defined by the ratio of the actual pressure and the free particle pressure, was determined numerically, and a Gaussian was fitted to it to a very good precision.

These results then were adapted for QCD. We used a simple model, where the masses of the hadronic resonances were distributed by Hagedorn distribution, and hadron melting was taken into account by a Gaussian width dependence, suggested by the model-scenarios. The width itself, in the simplest case, was just approximated by $\gamma^{2}(T)=\gamma_{0}^{2}+c T^{2}$. As a result we could fit the MC results for QCD thermodynamics (pressure and trace anomaly) up to $T_{c}^{\prime} \approx 200-250$ $\mathrm{MeV}$ with few parameters. We also determined the QGP pressure which, by lowering the temperature, vanishes in this temperature range.

What do these results tell us? We must say that, according to the statistical hadron picture combined with the spectral analysis of the hadron resonances, the hadron - QGP transition occurs in the temperature range of $T_{c}^{\prime} \sim 200$ $250 \mathrm{MeV}$. The reported crossover at the lower temperature $T_{c}=156 \mathrm{MeV}$ characterizes the hadron melting process: it is the starting point of rapid hadron melting. It can also be considered as a limiting temperature separating the regimes where the naive, free HRG model works from the one where the hadron width (more precisely the complete 
spectral function) becomes an important factor, and no uncorrelated hadrons are present any more. In this light the findings of [15] that the strange and non-strange sectors present different $T_{c}$, support the above picture, since the more massive hadrons may melt later. But for a good while the effective hadronic number of dof are still significantly higher than the number of dof of QGP. The hadronic pressure shrinks only at about $T_{c}^{\prime} \approx 200-250 \mathrm{MeV}$ temperature to that small values, that the QGP pressure becomes dominant.

Numerically it would be really challenging to find whether there is any signal of any phase transition in the temperature regime $T_{c}^{\prime} \sim 200-250 \mathrm{MeV}$. It is presently a much less studied temperature range, and from the existing data it is impossible to tell anything about it.

According to this proposal, the temperature range of $T \sim[156,250]$ should be treated by a correlated hadron gas model. In this model perturbative calculations are possible (provided one uses the full spectral function), while PQCD calculations are relevant from $T \sim 200-250 \mathrm{MeV}$. To test this picture, one should calculate other quantities, like thermodynamical observables at finite chemical potential, or transport coefficients. These findings could be relevant not just for collider physics, but, as in case of the chemical potential dependence, also for astrophysics.

\section{ACKNOWLEDGMENTS}

The author thanks long and instructive discussions with A. Patkós. He also acknowledges discussions with and important remarks from T.S. Bíró, Sz. Borsányi, S.D. Katz, P. Petreczky and Zs. Szép. This work is supported by the Hungarian Research Fund (OTKA) under contract No. K104292.

[1] for a review see: P. Braun-Munzinger, K. Redlich, and J. Stachel, In Hwa, R.C. (ed.) et al.: Quark gluon plasma 491-599, nucl-th/0304013.

[2] A. Andronic, P. Braun-Munzinger, K. Redlich and J. Stachel, Phys. Lett. B571, 36(2003) nucl-th/0303036

[3] F. Karsch, K. Redlich and A. Tawfik, Eur. Phys. J. C29, 549(2003) hep-ph/0303108

[4] P. Huovinen and P. Petreczky, Nucl. Phys. A 837, 26 (2010) arXiv:0912.2541 [hep-ph]].

[5] S. Borsanyi, G. Endrodi, Z. Fodor, A. Jakovac, S. D. Katz, S. Krieg, C. Ratti and K. K. Szabo, JHEP 1011, 077 (2010) arXiv:1007.2580 [hep-lat]].

[6] A. Bazavov, H. -T. Ding, P. Hegde, O. Kaczmarek, F. Karsch, E. Laermann, Y. Maezawa and S. Mukherjee et al., arXiv:1304.7220 [hep-lat].

[7] J. Beringer et al. (Particle Data Group), Phys. Rev. D86, 010001 (2012)

[8] Y. Aoki, G. Endrodi, Z. Fodor, S. D. Katz and K. K. Szabo, Nature 443, 675 (2006) hep-lat/0611014.

[9] R. Hagedorn, Nuovo Cim. Suppl. 3, 147 (1965);

[10] S. Datta, F. Karsch, P. Petreczky and I. Wetzorke, Phys. Rev. D 69, 094507 (2004) hep-lat/0312037.

[11] T. Umeda, K. Nomura and H. Matsufuru, Eur. Phys. J. C 39S1, 9 (2005) hep-lat/0211003.

[12] M. Asakawa and T. Hatsuda, Phys. Rev. Lett. 92, 012001 (2004) hep-lat/0308034.

[13] A. Jakovac, P. Petreczky, K. Petrov and A. Velytsky, Phys. Rev. D 75, 014506 (2007) hep-lat/0611017.

[14] P. Petreczky, J. Phys. Conf. Ser. 402, 012036 (2012) arXiv:1204.4414 [hep-lat]].

[15] R. Bellwied, S. Borsanyi, Z. Fodor, S. D. Katz and C. Ratti, arXiv:1305.6297 [hep-lat].

[16] U. W. Heinz and R. Snellings, arXiv:1301.2826 [nucl-th].

[17] D. B. Blaschke and K. A. Bugaev, Fizika B 13, 491 (2004) nucl-th/0311021.

[18] T. S. Biro and J. Zimanyi, Phys. Lett. B 650, 193 (2007) hep-ph/0607079.

[19] T. S. Biro, P. Levai, P. Van and J. Zimanyi, J. Phys. G 32, S205 (2006) hep-ph/0605274.

[20] Y. .B. Ivanov, J. Knoll and D. N. Voskresensky, Nucl. Phys. A 657, 413 (1999) hep-ph/9807351].

[21] Y. .B. Ivanov, J. Knoll and D. N. Voskresensky, Nucl. Phys. A 672, 313 (2000) nucl-th/9905028.

[22] A. Peshier and W. Cassing, Phys. Rev. Lett. 94, 172301 (2005) hep-ph/0502138.

[23] R.F. Dashen and R. Rajaraman, Phys.Rev. D10 (1974) 694; Phys.Rev. D10 (1974) 708

[24] H. Feshbach, Ann. Phys. 43, 110 (1967), L. Rosenfeld, Acta Phys. Polonica A38, 603 (1970).

[25] M. Svec, Phys. Rev. D 64, 096003 (2001) hep-ph/0009275].

[26] A. Jakovac, Phys. Rev. D 86, 085007 (2012) arXiv:1206.0865 [hep-ph]].

[27] W. Broniowski, W. Florkowski and L. Y. .Glozman, Phys. Rev. D 70, 117503 (2004) hep-ph/0407290.

[28] J. Noronha-Hostler, J. Noronha and C. Greiner, Phys. Rev. C 86, 024913 (2012) arXiv:1206.5138 [nucl-th]].

[29] J. Cleymans and D. Worku, Mod. Phys. Lett. A 26, 1197 (2011) arXiv:1103.1463 [hep-ph]].

[30] A. Jakovac, Phys. Rev. D 76, 125004 (2007) hep-ph/0612268.

[31] C. A. Dominguez, M. Loewe and J. C. Rojas, JHEP 0708, 040 (2007) arXiv:0707.2844 [hep-ph]].

[32] P. Colangelo, F. Giannuzzi and S. Nicotri, Phys. Rev. D 80, 094019 (2009) arXiv:0909.1534 [hep-ph]].

[33] F. Riek and J. Knoll, Nucl. Phys. A 740, 287 (2004) nucl-th/0402090. 